\title{
Paciente inmunosuprimido con fiebre $y$ radiografía con patrón miliar. Reporte de un caso.
}

Immunosupressed patient with fever and radiological images with miliary pattern: A case report.

SOTO ARQUIÍ̃IGO Leslie ${ }^{1}$, LEGUA LEIVAPedro ${ }^{1}$, GOTUZZO HERENCIA Eduardo ${ }^{1}$, ECHEVARRIAZARATE Juan $^{1}$, BUSTAMANTE RUFINO Beatriz ${ }^{1}$, MAGUIÑA VARGAS Ciro ${ }^{1}$, HERRERA ARANA Víctor ${ }^{1}$, MENDOZA TICONAAlberto ${ }^{1}$.

\section{SUMMARY}

We present the case of a patient proceeding from the jungle of Peru with diagnosis of infection for the Human Immunodeficiency Virus (HIV) with respiratory symptoms, fever and a chest X-ray with milliary pattern. Because Tuberculosis is a prevalent disease in our country and the most frequent in patients with HIV, he received antituberculous treatment. Nevertheless, the historical information of having been in endemic zone of mycosis in some moment of his life, associated with laboratory examinations, allowed the diagnosis of Disseminated histoplasmosis, as product of the reactivation of a latent infection that appears when the level of immunity gets depressed like in infected patients by the HIV. The history of diseases, trips and previous residences is a fundamental information to establish a good approximation diagnosis in patients with HIV (Rev Med Hered 2005;16:218-222).

KEY WORDS: Disseminated histoplasmosis, HIV, fever, milliary pattern.

\section{RESUMEN}

Se presenta el caso de un paciente procedente de la selva de Perú con diagnóstico de infección por el Virus de Inmunodeficiencia Humana (VIH) con cuadro respiratorio alto, fiebre y que presentó una radiografía con patrón miliar. Por ser la Tuberculosis una enfermedad prevalente en nuestro país e infección mas frecuente en pacientes con VIH recibió tratamiento anti tuberculoso. Sin embargo, el dato histórico de haber estado en zona endémica de micosis en algún momento de su vida, asociado a exámenes de laboratorio, permitió el diagnóstico de Histoplasmosis diseminada, como producto de la reactivación de una infección latente que se manifiesta cuando el nivel de inmunidad se deprime como ocurre en los paciente infectados por el VIH. La historia de enfermedades, viajes y residencias previas es información fundamental para establecer una buena aproximación diagnóstico en pacientes con VIH. (Rev Med Hered 2005;16:218-222).

PALABRAS CLAVE: Histoplasmosis diseminada, VIH, fiebre, patrón miliar.

Instituto de Medicina Tropical Alexander von Humboldt. Universidad Peruana Cayetano Heredia. Departamento de Enfermedades Infecciosas Tropicales y Dermatológicas del Hospital Nacional Cayetano Heredia. 
Se presenta el caso de un paciente varón de 38 años, soltero, natural de Madre de Dios, procedente de Lima, con diagnostico de infección por el Virus de Inmunodeficiencia Humana (VIH) desde 1995, que al momento de inicio del proceso actual no había recibido tratamiento profiláctico ni antirretroviral. Fue admitido con una historia de 3 meses de enfermedad caracterizada por fatiga, debilidad generalizada y baja de peso progresiva. Un mes antes de hospitalizarse se agrega fiebre $\left(38^{\circ} \mathrm{C}\right)$. Acude al Hospital Nacional Cayetano Heredia $(\mathrm{HNCH})$ donde le solicitan exámenes de esputo para la tinción Ziehl Neelsen cuyos resultados fueron negativos en 3 oportunidades, PPD siendo el resultado negativo y una radiografía de tórax en la que se encontró un patrón intersticial micronodular sugestivo de tuberculosis (TBC) pulmonar miliar (Figura $\mathrm{N}^{\circ} 1$ ).

Siendo la diseminación miliar la forma más frecuente de compromiso pulmonar tuberculoso entre los pacientes que sufren de SIDA, le indicaron tratamiento con isoniacida, rifampicina, pirazinamida y etambutol a dosis estándar. Durante el tratamiento el paciente presentó náuseas, vómitos, tos no productiva y persistencia de la fiebre $\left(39^{\circ} \mathrm{C}\right)$. Al no haber mejoría fue hospitalizada.
El paciente presentó hiporexia, pobre ingesta de alimentos y disminución de peso en $17 \mathrm{~kg}$. Como dato epidemiológico informó haber residido en Puerto Maldonado (Selva), Arequipa (Sierra), Japón y desde hace 11 años en Lima. No existía historia de consumo de drogas ilícitas, alcohol y tabaco.

Al examen físico de ingreso: PA: 120/70 FC: $120 \mathrm{x}^{\prime}$ FR: $29 x^{\prime} \mathrm{T}: 38,5^{\circ} \mathrm{C}$ Peso: $57 \mathrm{Kg}$. No se observó lesiones dérmicas. El fondo de ojo fue descrito como normal. Ganglios aumentados de tamaño a nivel cervical y axilar derecho siendo los de mayor diámetro de $2 \times 2 \mathrm{~cm}$., muguet oral. El examen pulmonar evidenció polipnea, amplexación disminuida y crépitos difusos en ambos campos pulmonares. En el abdomen se encontró hepatomegalia y el resto del examen físico fue no contributorio.

Como parte de la valoración de este paciente con patrón miliar, fiebre persistente y falla a la terapia anti TBC se obtuvo del plan de estudio: $\mathrm{CD}^{+} \mathrm{T}$ : 18 células/ $\mathrm{mm}^{3}$, Pruebas de función hepática se encontró fosfatasa alcalina: 1031 UL, DHL: 474 UL, con bilirrubinas, TGO y TGP normales. Los estudios serológicos de VDRL, HBsAg, Anti HBc, HTLV-1 fueron no reactivos.

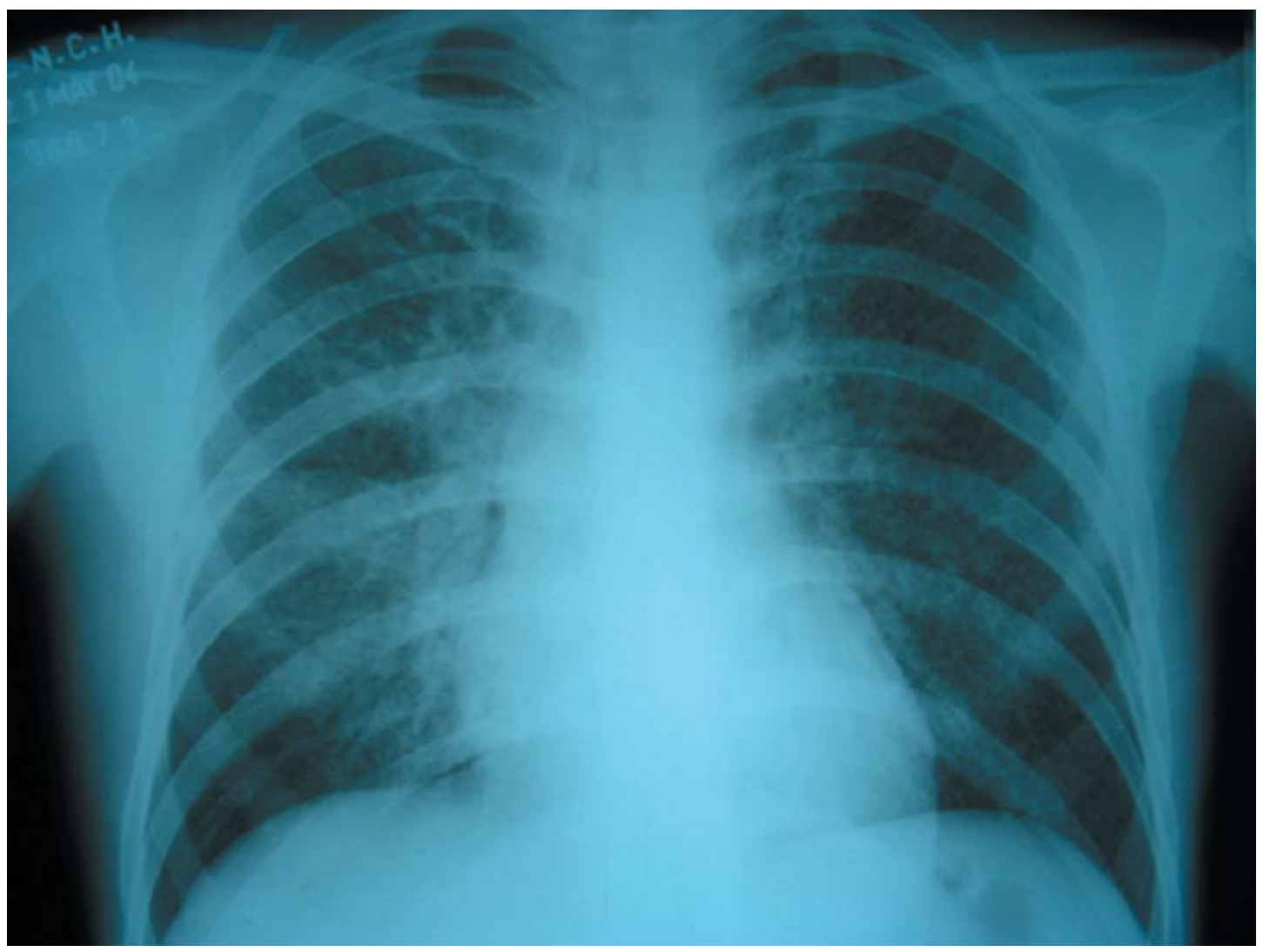

Figura No1. Radiografia de torax mostrando patrón intersticial micronodular. 
Aglutinaciones para fiebre tifoidea y brucelosis negativas. Anemia moderada con hematocrito en $29 \%$, leucocitos en rangos normales sin desviación izquierda y plaquetas con recuento normal. Estudio de esputo: Ziehl Neelsen: Negativo, Giemsa: Negativo para quistes de PCP; Serología: Inmunodifusión para H. Capsulatum y P. brasiliensis: Negativos, Látex para C. neoformans: Negativo.

Ecografía abdominal: Hepatoesplenomegalia, pequeñas adenopatías intercavaorticas, que miden 14 y $16 \mathrm{~mm}$, otras menores de $10 \mathrm{~mm}$ respectivamente.

Cultivos: Hemocultivo, Urocultivo y Coprocultivo negativos.

Biopsia de Hueso: Hueso con escaso tejido medular evaluable en la que se observa una celularidad del $20 \%$ con respecto al tejido adiposo. Presencia de las 3 series con arresto de la maduración mieloide. No se observaron granulomas.

Biopsia de ganglio cervical: Proceso inflamatorio crónico granulomatoso con presencia de estructuras levaduriformes en las coloraciones H-E y PAS sugerente de criptococosis a descartar histoplasmosis.

Aspirado de Médula Ósea (AMO): Pancitopenia, anemia moderada, normocítica, normocrómica, de causa multifactorial. No células neoplásicas; Giemsa del AMO: Levaduras pequeñas. Cultivo BK: Negativo, Mielocultivo: Histoplasma capsulatum.

Diagnóstico: Histoplasmosis diseminada.

\section{DISCUSIÓN}

La histoplasmosis es una enfermedad causada por el hongo Histoplasma capsulatum var capsulatum que crece en el suelo y es enriquecido con la excreta de aves y murciélagos que aceleran la esporulación.

Es conocido que un tercio de las muertes relacionadas al SIDA son debidas a tuberculosis (1), y que los pacientes coinfectados desarrollan TBC activa a tasas de 5 a 16/100 personas año (2). Debemos sospechar esta activación en sujetos que presentan fiebre, baja de peso con o sin manifestaciones respiratorias, y radiografía de tórax que muestre un patrón miliar. La tinción de Ziehl Neelsen de esputo suele ser positiva en no más del $60 \%$ de los pacientes, y el PPD tiene elevada proporción de falsos negativos $(65 \%)$ en el grupo de pacientes con infección por el HIV y TBC activa (3). Siendo la TBC una enfermedad prevalente en nuestro país y, además, la infección más frecuente en pacientes con infección por el VIH, la indicación de inicio de la terapia antituberculosa en este paciente fue apropiada. Sin embargo, debemos ser concientes de que la presencia de un patrón miliar y fiebre se presenta en otras infecciones entre las que se incluye a la neumonía por Pneumocystis jiroveci, enfermedad por el citomegalovirus, criptococosis miliar, histoplasmosis, coccidiodomicosis y, menos frecuentemente, en la neumonía intersticial linfocitaria, toxoplasmosis y la infección por Haemophilus influenzae.

La respuesta esperada a la terapia anti $\mathrm{TBC}$ para éste y otros pacientes con diagnóstico de tuberculosis y SIDA, es que la curva febril ceda entre el día 7 y 14 del tratamiento. La persistencia de fiebre más allá de este tiempo nos sugiere resistencia al tratamiento anti TBC u otra causa de la fiebre, entre las que se deben considerar otras infecciones oportunistas o tumores (4).

En los estudios de fiebre de origen desconocido en pacientes con VIH, se menciona entre los diagnósticos más comúnmente hallados a la infección por Mycobacterium avium diseminado (31\%), neumonía por Pneumocystis jiroveci (13\%), infección por citomegalovirus $(11 \%)$, histoplasmosis diseminada (7\%), y linfoma (7\%) (5).

Los pacientes con SIDA desarrollan enfermedades por agentes oportunistas por la reactivación de una infección antigua o, en su defecto, por exposición reciente al agente. La historia de enfermedades, viajes y residencias previas es información fundamental para establecer una buena aproximación al diagnóstico. El nivel de inmunidad (recuento de $\mathrm{CD}^{+} \mathrm{T}$ ) nos orientará hacia los agentes oportunistas más frecuentes, ya que existe un patrón de frecuencia de infecciones relacionado al nivel de compromiso del sistema inmunológico (6).

La distribución es mundial, siendo prevalente en ciertas partes de Norteamérica y América del Centro (7). En Sudamérica, se encuentra mayormente en Brasil, Colombia, Argentina (8), y en nuestro país se encuentra en toda la Selva peruana siendo zonas endémicas Tingo María, Pucallpa e Iquitos entre otras (9).

La histoplasmosis en pacientes con VIH se presenta en forma diseminada en el $95 \%$ de los casos, usualmente aparece en sujetos con recuento de $\mathrm{CD} 4^{+} \mathrm{T}$ menor de 200 células $/ \mathrm{mm}^{3}$, pero la gran mayoría de las veces con $\mathrm{CD} 4+\mathrm{T}$ : $<75$ células $/ \mathrm{mm}^{3}$. La incidencia global es de $0,9 \%$, en áreas endémicas se presenta entre $2 \%$ a 
$5 \%$ pero en zonas con alta endemicidad como Indiana (EE UU) es del 27\%. Histoplasmosis diseminada fue identificada en autopsias en $8 \%$ de pacientes con SIDA en Brasil y $44 \%$ en Venezuela (8), por lo que su presencia es más común de la que se pensaba.

La reactivación de la infección latente es más probable que la infección exógena. Existen indicios de reactivación de histoplasmosis porque personas nacidas en áreas endémicas pero que migran y no regresan a su lugar de origen, o personas que viven en zonas no prevalentes, pero que tienen el dato histórico de haber visitado tiempo atrás zonas endémicas, presentan la enfermedad cuando desarrollan inmunosupresión, meses ó años después de haber dejado la zona de exposición (10). En el caso de nuestro paciente, ayudó a orientar el diagnóstico el lugar de nacimiento y residencia, Puerto Maldonado (Madre de Dios), en donde vivió 10 años. Por esta razón es fundamental indagar en pacientes como el nuestro, información sobre historia de residencias y viajes previos al desarrollo de la enfermedad.

Usualmente se presenta fiebre, fatiga y baja de peso como ocurrió en nuestro paciente y cerca del $50 \%$ presenta tos y disnea. Al examen físico se encuentra hepatoesplenomegalia y linfadenopatías, hallazgos que encontramos, no hubo lesiones dérmicas ya que son menos frecuentes $(10 \%)$, ni síntomas neurológicos $(10 \%)$ o gastrointestinales (10\%). Menos del $10 \%$ se presentan con síntomas similares a una sepsis, y la mitad de ellos mueren dentro de la primera semana de diagnóstico (11). Es característica la presentación subaguda de 1 a 3 meses de evolución.

El hallazgo radiográfico obtenido no es patognomónico, ya que es anormal entre el 50\% a 75\% de pacientes y se encuentra infiltrado reticulonodular o intersticial difuso, incluso cavidades y patrón normal. La linfadenopatía mediastinal se encuentra en $<10 \%$ de los casos.

La presencia de levaduras en la tinción Giemsa en médula ósea ayudó en el diagnóstico, pudiéndose haber utilizado también las tinciones de Gram, H-E, Wright siendo la mejor la tinción de Gomori. Se sabe que el mejor espécimen para la detección se obtiene de la médula ósea hasta $50-75 \%$ de los pacientes (6).

La detección de antígeno es mas útil en pacientes con infección diseminada, ayudan en el diagnóstico antes que los cultivos sean positivos e incluso antes que aparezcan los anticuerpos. Se puede realizar en la orina o en el suero con una sensibilidad de $90 \%$ y $85 \%$ respectivamente (12).
Los anticuerpos en títulos altos se desarrollan dentro de las 4 a 6 semanas de infección aconsejándose usar las pruebas de inmunodifusión y fijación del complemento, sin embargo, son menos sensitivas en pacientes inmunosuprimidos, los títulos son mas bajos y las pruebas serológicas positivas, pueden ser de infecciones previas o de otra enfermedad, lo que explicaría que se haya obtenido un resultado negativo en el paciente.

Los cultivos de sangre, aspirado de médula ósea y secreciones respiratorias pueden ser positivas en más del $85 \%$ de los casos, lamentablemente el cultivo demora de 2 a 4 semanas (6), lo que pudiera retrasar el diagnóstico, por lo que la sospecha diagnóstica por lugar de procedencia, hallazgos en las tinciones Giemsa o Wright de las muestras obtenidas, y estado de la inmunidad nos ayudan a implementar un tratamiento rápido.

La prueba dérmica de histoplasmina no es útil para el diagnóstico, ya que en un individuo con VIH esta alterada la inmunidad mediada por células con la infección avanzada.

El tratamiento instaurado en nuestro paciente fue anfotericina $B$ endovenoso seguido por terapia supresiva de itraconazol por vía oral hasta la fecha, a dosis standard. La respuesta de la terapia es variable se utiliza anfotericina B (68\%-91\%), itraconazol (85\%-100\%), ketoconazol (9\%-70\%) y fluconazol (74\%-86\%). La mortalidad sin tratamiento es de $80 \%$, el tratamiento con anfotericina $\mathrm{B}$ reduce a $<25 \%$. En pacientes con SIDA se recomienda terapia de mantenimiento con itraconazol de por vida, sin embargo en estudios actuales se sugeriría la descontinuación de tratamiento cuando el recuento celular de linfocitos CD4+ es mayor de 150 células $/ \mathrm{mm}^{3}$ (13).

Se recomienda profilaxis con Itraconazol $200 \mathrm{mg}$ qd en personas con el VIH y CD4 $<150 \mathrm{cel} / \mathrm{mm}^{3}$ que residen en lugares con tasas de histoplasmosis que excede los 10 casos por 100 pacientes año, en un estudio controlado se redujo la ocurrencia de histoplasmosis y criptococosis en $70 \%$ con el uso de itraconazol. Todos los pacientes con inmunosupresión deben evitar todo contacto con áreas con prevalencia de esta entidad.

Al momento del reporte, el paciente se encuentra asintomático, ha subido 15 kilos de peso, está recibiendo profilaxis para histoplasmosis y ha iniciado tratamiento antiretroviral.

El presente caso nos muestra un paciente con histoplasmosis diseminada con compromiso pulmonar, 
hepático, esplénico y de ganglios linfáticos que simulaba un caso de tuberculosis miliar, sin embargo hay que tener en cuenta la procedencia de los pacientes ya que micosis profundas se reactivan cuando el nivel de inmunidad se deprime como ocurre en los pacientes infectados por VIH.

\section{Correspondencia:}

Leslie Soto Arquinigo

Correo electrónico: 1soto@viabcp.com

\section{REFERENCIAS BIBLIOGRÁFICAS}

1. World Health Organization. Groups at risk: WHO Report on the Tuberculosis Epidemic Geneva: World Health Organization; 1996.

2. Dolin R, Masur H, Saag M. AIDS Therapy. Second edition. Edinburgh: Churchill Livingstone; 1999: 864

3. Johnson MP, Coberly JS, Clermont HC, Chaisson RE, Davis HL, Losikoff P, Ruff AJ, Boulos R, Halsey NA. Tuberculin skin test reactivity among adults infected with human immunodeficiency virus. J Infect Dis 1992; 166(1):194-8.

4. Busillo CP, Lessnau KD, Sanjana V, Soumakis S, Davidson M, Mullen MP, et al. Multidrug resistant Mycobacterium tuberculosis in patients with human immunodeficiency virus infection. Chest 1992; 102: 797-801

5. Armstrong W, Katz J, Kazanjian P. Human Immunodeficiency Virus-Associated fever of unknown origin: A study of 70 patients in the United States and review. Clin Infect Dis 1999;28:341-5.

6. Bartlett J, Gallant J. Medical management of HIV Infection. Baltimore MD: John Hopkins University School of Medicine;2004.

7. Wheat LJ, Kauffman CA. Histoplasmosis. Infect Dis Clin North Am 2003;17 (1):1-19.

8. Murillo J, Castro KG. HIV infection and AIDS in Latin America. Infect Dis Clin North Am 1994; 8:111.

9. Morales P, Paz E, Medina J, Yoza M, Narvaez J. Histoplasmosis diseminada en niños: Reporte de dos casos. Diagnóstico 1996; 35 (3): 39-45.

10. Keath EJ, Kobayashi GS, Medoff G. Typing of histoplasma capsulatum by restriction fragment length polymorphisms in a nuclear gene. J Clin Microbiol 1992 (8):2104-7.

11. Wheat LJ, Connoly-Stringfield PA, Baker RL, et al. Disseminated histoplasmosis in the acquired immune deficiency syndrome: clinical findings, diagnosis and treatment, and review of literature. Medicine 1990; 69:361.

12. Wheat J. Current Diagnosis of histoplasmosis. Trends in Microbiology 2003; 11 (10):10488-494.

13. Goldman M, Zackin R, Fichtenbaum CJ, Skiest DJ, Koletar SL, Hafner R, Wheat LJ, Nyangweso PM, Yiannoutsos CT, Schnizlein-Bick CT, Owens S, Aberg JA, AIDS Clinical Trials Group A5038 Study Group. Safety of discontinuation of maintenance therapy for disseminated histoplasmosis after immunologic response to antiretroviral therapy. Clin Infect Dis 2004 ;38(10):1485-9.

Recibido: 09/11/04

Aceptado para publicación: 11/04/05 